\title{
Non-perturbative renormalization in domain-wall QCD by Schrödinger functional scheme *
}

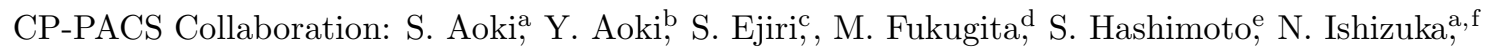
Y. Iwasaki, T. Izubuchi, K. Kanaya, T. Kaneko, Y. Kuramashi, M. Okawa, Y. Taniguchi, A. Ukawa ${ }^{\text {a,f }}$ and T. Yoshiéa,f

${ }^{a}$ Institute of Physics, University of Tsukuba, Tsukuba, Ibaraki 305-8571, Japan

${ }^{\text {b} R I K E N ~ B N L ~ R e s e a r c h ~ C e n t e r, ~ B r o o k h a v e n ~ N a t i o n a l ~ L a b o r a t o r y, ~ U p t o n, ~ N Y ~ 11973, ~ U S A ~}$

${ }^{\mathrm{c}}$ Department of Physics, University of Wales Swansea, Singleton Park, Swansea SA2 8PP, UK

${ }^{\mathrm{d}}$ Institute for Cosmic Ray Research, University of Tokyo, Kashiwa 277-8582, Japan

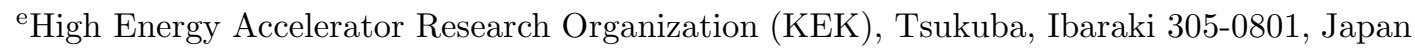

${ }_{\mathrm{f}}^{\mathrm{f}}$ Center for Computational Physics, University of Tsukuba, Tsukuba, Ibaraki 305-8577, Japan

'shysics Department, Brookhaven National Laboratory, Upton, NY 11973, USA

\begin{abstract}
We formulate and numerically test the Schrödinger functional scheme for domain-wall QCD. We then apply it to a non-perturbative calculation of the renormalization factors for vector and axial-vector currents in quenched domain-wall QCD with plaquette and renormalization group improved gauge actions at $a^{-1} \simeq 2 \mathrm{GeV}$.
\end{abstract}

\section{Introduction}

Recent calculations with domain-wall QCD (DWQCD) have shown that the good chiral property of domain-wall fermions leads to good scaling behavior of physical observables such as quark mass and $B_{K}[$ [1]. Aside from the quenched approximation, the use of perturbative renormalization factors is the largest source of the uncontrolled systematic errors in these calculation. Non-perturbative renormalization is required to reduce the total error to a few percent except for quenching, and we decide to employ the Schrödinger functional(SF) scheme[2], which is a gauge-invariant and finite volume method, toward this goal.

In this report we formulate the SF scheme for DWQCD and calculate the renormalization factors for vector and axial-vector currents as a first step to obtain the renormalization factors for the quark mass and $B_{K}$.

\footnotetext{
*Poster presented by S. Aoki
}

\section{Schrödinger Functional in DWQCD}

We consider DWQCD[3] on an $L^{3} \times T \times N_{s}$ lattice. The SF boundary condition for the domain-wall fermion is given by $\psi\left(\vec{x}, x_{0}=0, s\right)=$ $P_{+} P_{L}(s) \rho(\vec{x}), \bar{\psi}\left(\vec{x}, x_{0}=0, s\right)=\bar{\rho}(\vec{x}) P_{R}(s) P_{-}$, $\psi\left(\vec{x}, x_{0}=T, s\right)=P_{-} P_{L}(s) \rho^{\prime}(\vec{x})$ and $\bar{\psi}\left(\vec{x}, x_{0}=\right.$ $T, s)=\bar{\rho}^{\prime}(\vec{x}) P_{R}(s) P_{+}$. Here $\rho, \bar{\rho}$ are the boundary quark fields, $P_{L}(s)=P_{L} \delta_{s, 1}+P_{R} \delta_{s, N_{s}}$ and $P_{R}(s)=P_{R} \delta_{s, 1}+P_{L} \delta_{s, N_{s}}$ with $P_{R / L}=\frac{1}{2}\left(1 \pm \gamma_{5}\right)$ and $P_{ \pm}=\frac{1}{2}\left(1 \pm \gamma_{0}\right)$.

We follow the definitions and notations of ref. [1] for the calculation of the renormalization factors.

\section{Tests of the formulation}

Since the SF boundary condition in time for $\rho=\bar{\rho}=0$ is identical to the Shamir's domainwall(Dirichlet) boundary condition in the fifth direction[5], extra zero modes appear near $x_{0}=0$ and $T$. We check whether these unwanted zero modes induce extra contribution to the low en- 
2

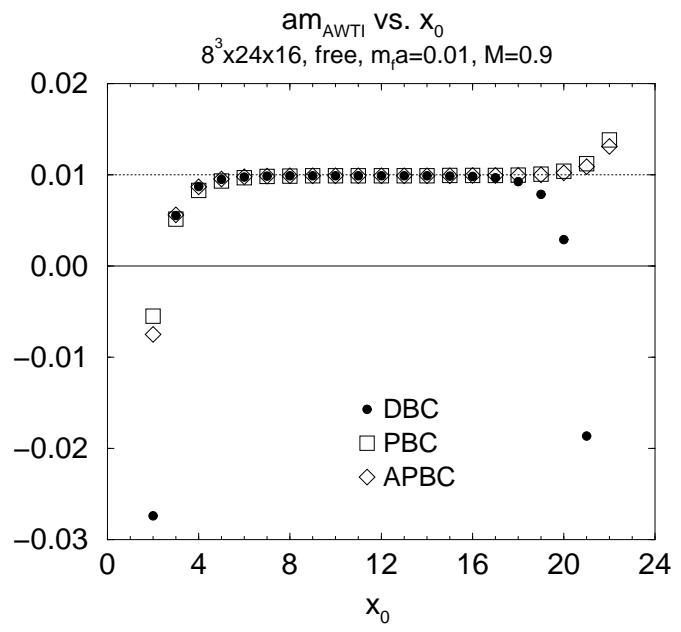

Figure 1. $a m_{\mathrm{AWTI}}$ as a function of $x_{0}$ with Dirichlet(solid circles), periodic(open squares) and antiperiodic(open diamonds) boundary conditions.

ergy observables at $0 \ll x_{0} \ll T$. Here we consider the quark mass, $a m_{\mathrm{AWTI}}$, defined through the axial Ward-Takahashi identity(AWTI). In Fig. 1, we plot $a m_{\text {AWTI }}$ for free theory as a function of $x_{0}$ with Dirichlet, periodic and antiperiodic boundary conditions at the bare quark mass $m_{f} a=0.01$, on an $8^{3} \times 24 \times 16$ lattice, with the domain-wall height $M=0.9$. The dependence of $a m_{\text {AWTI }}$ on the boundary condition, which is visible near the boundaries, disappears away from them. Therefore we conclude that the extra zero modes associated with the Dirichlet boundary condition gives negligible effects to the determination of the renormalization factors evaluated at $x_{0} \simeq T / 2$. Further analytic investigations on this problem for the free case will be given elsewhere[6].

In Fig. 2, $a m_{\mathrm{AWTI}}$ for the SF scheme is plotted in quenched DWQCD at $\beta=6.0$ of the plaquette action. The $x_{0}$ dependence is also weak away from the boundaries. Interestingly $m_{\mathrm{AWTI}}$ is nonzero even at $m_{f} a=0$, and becomes smaller for larger $N_{s}$. Moreover the value is consistent with $m_{5 q}$, the explicit breaking of chiral symmetry calculated from the conserved axial vector current of DWQCD[7]. Therefore $m_{\text {AWTI }}$ for the SF scheme may be a better alternative as the measure of explicit chiral symmetry breaking in DWQCD, since it can be calculated directly at $m_{f} a=0$ with

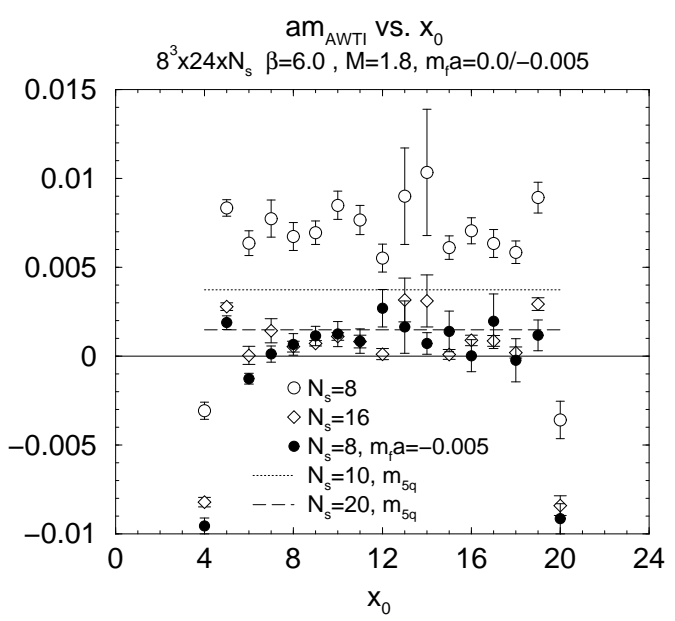

Figure 2. $a m_{\mathrm{AWTI}}$ as a function of $x_{0}$ at $\beta=6.0$ on an $8^{3} \times 24 \times N_{s}$ lattice at $m_{f} a=0$ (open symbols) and -0.005 (solid circles), together with $m_{5 q}$ [7].

much less computational cost. Note also that the large explicit breaking in $m_{\text {AWTI }}$ at $N_{s}=8$ (open circles) is well compensated if one takes a negative quark mass of $m_{f}=-0.005$ (filled circles). Hence the domain-wall fermion at $N_{s} \neq \infty$ may be considered as a highly improved Wilson fermion [8].

The non-perturbative renormalization factors for vector and axial-vector currents are defined by $Z_{V}\left(1+b_{V} m_{f} a\right)=f_{1} / f_{V}\left(x_{0}\right)$ and $Z_{A}^{2}=$ $2 f_{1} / f_{A A}\left(x_{0}, x_{0}^{+}, x_{0}^{-}\right)$, where we fix $x_{0}^{ \pm}=T / 2 \pm$ $T / 4$ and put $m_{f} a=0$ for $Z_{A}$. In Fig. 3, $Z_{V}$ and $Z_{A}$ are plotted as a function of $x_{0}$ at $\beta=6.0$. Similar to the case of $a m_{\text {AWTI }}$ a plateau is seen away from the boundaries. The relation $Z_{V}=Z_{A}$, exactly valid in perturbation theory, is satisfied within 1-2\%. Moreover the magnitude almost agrees with the mean-field(MF) improved one-loop value. We also observe that $Z_{V}$ is insensitive to boundary parameters such as the 2-loop boundary counter-terms for gauge fields and the parameter $\theta$ of the twisted boundary condition for quarks.

\section{Results}

We have calculated $Z_{V}$ and $Z_{A}$ in quenched DWQCD at $a^{-1} \simeq 2 \mathrm{GeV}$ with the plaquette action $(\beta=6.0)$ and with the renormalization $\operatorname{group}(\mathrm{RG})$ improved action $(\beta=2.6)$ on an $8^{3} \times 16 \times 16$ lattice with $m_{f} a=0$ for 


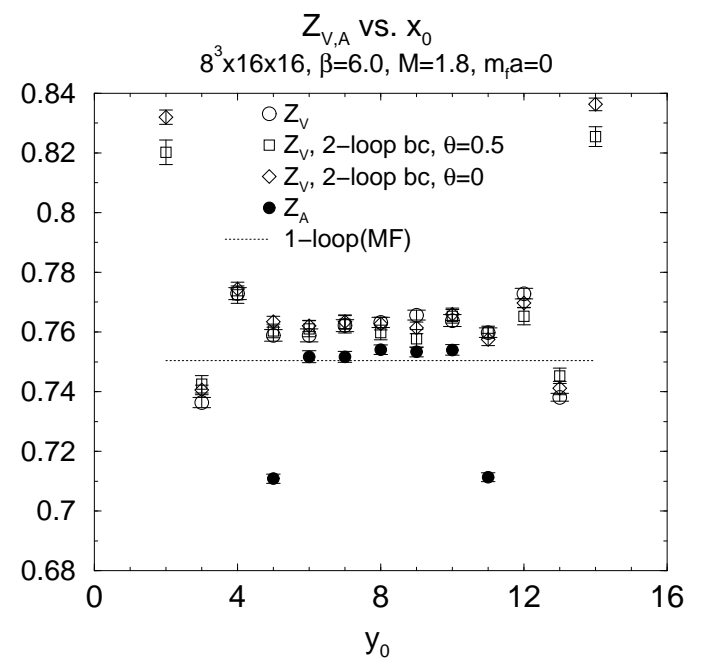

Figure 3. $Z_{V, A}$ as a function of $x_{0}$ at $\beta=6$ on an $8^{3} \times 16 \times 16$ lattice with $M=1.8$ and $m_{f} a=$ 0 . We compare the results from the boundary counter-terms at tree-level(circles) with those at 2-loop(squares and diamonds) as well as those at $\theta=0$ with that at $\theta=0.5$ (squares).

$M=1.0 \sim 2.2$. The results are summarized in Fig. 目, where $Z_{V}$ and $Z_{A}$ are plotted as a function of $M$, together with one-loop perturbative estimates with and without MF improvement. For both gauge actions, $Z_{V} \simeq Z_{A}$ holds, and they show a minimum at $M \simeq 1.7$ for the plaquette action or $M \simeq 1.6$ for the $\mathrm{RG}$ action. Perturbative estimates without MF improvement fail, particularly for the plaquette action for which the curve can not be placed in the figure. The agreement becomes much better with MF improvement for both actions.

\section{Future directions}

We are encouraged with the present results to proceed to an extension of the present work to scale-dependent cases such as quark masses and four-quark operators needed for $B_{K}$.

S.A. thanks Profs. M. Lüscher, P. Weisz and H. Wittig for useful discussion. This work is supported in part by Grants-in-Aid of the Ministry of Education (Nos.11640294, 12014202, 12304011, 12640253, 12740133, 1364026013135204 ).
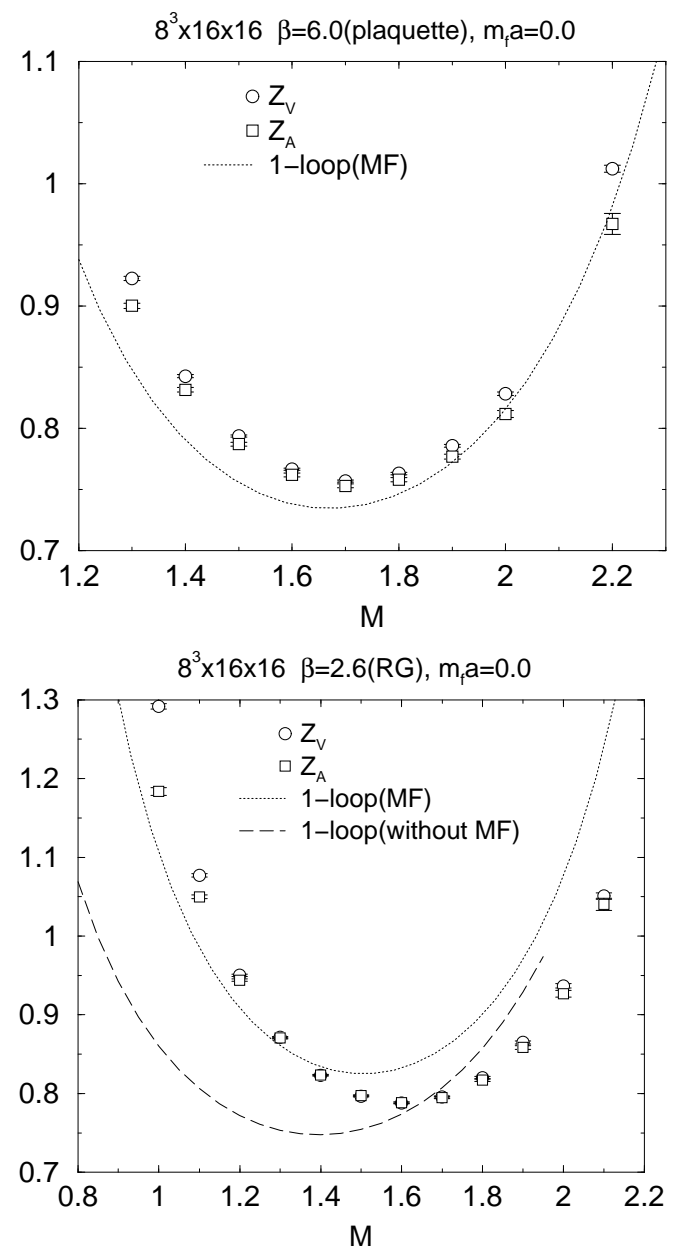

Figure 4. $Z_{V}$ and $Z_{A}$ vs $M$ for plaquette(upper) and $\mathrm{RG}$ (lower) gauge actions.

\section{REFERENCES}

1. CP-PACS Collaboration: A. Ali Khan, et al., hep-lat/0105020.

2. ALPHA collaboration: M. Lüshcer et al., Nucl. Phys. B384 (1992) 168;B478 (1996) 365.

3. V. Furman and Y. Shamir, Nucl. Phys. B439 (1995) 54.

4. ALPHA collaboration: M. Lüshcer et al. , Nucl. Phys. B491 (1997) 344; S. Capitani et al., Nucl. Phys. B544 (1999) 669.

5. Y. Shamir, Nucl. Phys. B406 (1993) 90.

6. S. Aoki and Y. Kikukawa, work in progress.

7. CP-PACS Collaboration: A. Ali Khan, et al., Phys. Rev. D63 (2001) 114504.

8. S. Aoki et al., Phy. Rev. D62 (2000) 094502. 